\title{
Antimicrobial stewardship in the Western Cape: A situational analysis of existing facility-level initiatives
}

\author{
S M Peters, ${ }^{1,2,3}$ MB ChB, BA, MMed (Public Health Medicine), FCPHM (SA); S Sheik, ${ }^{2,4}$ MB ChB, MMed (Public Health Medicine), FCPHM (SA); \\ J L Werner, ${ }^{2,3}$ MB ChB; M-A Davies, ${ }^{2,3}$ MB ChB, MMed (Public Health Medicine), FCPHM (SA), PhD; \\ B Willems, ${ }^{2,4} \mathrm{MB}$ ChB, MMed (Public Health Medicine), FCPHM (SA) \\ ${ }^{1}$ Groote Schuur Hospital, Cape Town; and Department of Health, Western Cape Government, Cape Town, South Africa \\ ${ }^{2}$ Health Impact Assessment Unit, Department of Health, Western Cape Government, Cape Town, South Africa \\ ${ }^{3}$ School of Public Health and Family Medicine, Faculty of Health Sciences, University of Cape Town, South Africa \\ ${ }^{4}$ Department of Global Health, Faculty of Medicine and Health Sciences, Stellenbosch University, Cape Town, South Africa
}

Corresponding author: S M Peters (shrikant.peters@westerncape.gov.za)

\begin{abstract}
Background. Antimicrobial resistance (AMR) is a growing problem worldwide. With the current occurrence of pan-resistant bacterial infections and a paucity of novel antimicrobials in development, the world has entered a post-antibiotic era, in which previously treatable, common infections can become fatal. Antimicrobial stewardship (AMS), defined as 'co-ordinated interventions to ensure appropriate and rational use of antimicrobials', aims to decrease rates of AMR.

Objectives. To co-ordinate AMS in Western Cape Province. The National Department of Health (NDoH) has identified AMS as a key strategic objective, and the Western Cape has formed a provincial AMS committee. However, not much is known regarding current AMS activities in health facilities in the province.

Methods. A self-administered, email questionnaire was sent to specific staff at all district, regional and tertiary hospitals in the 6 health districts of the Western Cape - 47 facilities in total, of which 35 (74.4\%) responded. Respondents included pharmacists, managers, doctors, nurses, infection prevention and control practitioners, as well as quality assurance practitioners. The number of facilities implementing AMS were determined, as well as the composition of AMS committees and the nature and frequency of team activities. Barriers to facilitylevel AMS were explored. Support and outreach activities were assessed, as well as facilities' needs and expectations of the provincial AMS committee.

Results. Approximately half of all responding hospitals ( $n=19 ; 54.3 \%)$ had active AMS committees. Double the proportion of metropolitan $(83.3 \%)$ than rural facilities $(39.1 \%)$ had committees. Stewardship activities included antimicrobial prescription chart reviews and audits, AMS ward rounds, antimicrobial restriction policies and training. Most committees included a pharmacist and an infection prevention and control practitioner. More than a third of hospitals (36.1\%) did not review their antimicrobial consumption data on a regular basis. Just over half of the hospitals $(n=18 ; 51.4 \%)$ did not review AMR patterns.

Conclusions. Despite the need for effective AMS, there is limited information on AMS in South Africa. Most assistance is required in rural areas and smaller hospitals with low numbers of staff and greater numbers of transient rotating junior staff. Information management support, multidisciplinary teamwork and clinical governance are required to enable regular and ongoing AMS in facilities. Rural and smaller facilities require greater support to establish effectively functioning AMS committees.
\end{abstract}

S Afr Med J 2021;111(5):421-425. https://doi.org/10.7196/SAMJ.2021.v111i5.14645

Emerging antimicrobial resistance (AMR) worldwide, coupled with a paucity of novel antimicrobials in the drug development pipeline, has led to a post-antibiotic era in which previously treatable common infections can lead to death. ${ }^{[1]}$ It is estimated that, if left unchecked, AMR will be responsible for 10 million deaths annually and a decline in the global gross domestic product by $2-3.5 \%$ by $2050 .{ }^{[2]}$

Inappropriate antimicrobial management and consumption accelerate the pace and spread of AMR; therefore, the need for prioritising antimicrobial stewardship (AMS) in combating AMR. AMS focuses on appropriate and rational use of antimicrobials, and stewardship programme models vary worldwide. ${ }^{[3]}$ Such programmes are often implemented by multidisciplinary teams ${ }^{[3]}$ and may include a combination of restrictive and persuasive interventions. Restrictive interventions limit clinician freedom to prescribe certain antimicrobials and may include formulary restriction, prescription authorisation by designated specialists (e.g. infectious disease clinicians), automatic stop-orders and therapeutic substitutions. Persuasive interventions may take the form of education or training, e.g. on ward rounds or outreach visits, and may also include reminders, audit and feedback on prescribing behaviour. ${ }^{[4,5]}$

In the local context, AMS has been shown to be effective in reducing antimicrobial consumption, without negatively impacting patient outcomes ${ }^{[5-7]}$ and the implementation of such programmes has been reported to reduce pharmaceutical costs. ${ }^{[4,6]}$ Furthermore, the National Department of Health $(\mathrm{NDoH})$ has identified AMS as one of three strategic objectives in its Antimicrobial Resistance National Strategy Framework published in 2019. ${ }^{[8]}$

AMS champions are defined in the government's national guidelines as being enthusiastic healthcare workers who 'play vital advocacy roles to gather support, generate awareness and overcome barriers to implementation of antimicrobial stewardship activities'. The document envisions such champions to be formally appointed by provincial heads of department, and to be given the authority, mandate, resources and training to implement AMS activities. ${ }^{[9]}$

A provincial AMS committee was formed by the Department of Health, Western Cape Government, in 2013 in response to an 
outbreak of carbapenem-resistant Enterobacteriaceae at public sector hospitals in the region. Key roles of this committee include the provision of support, training and leadership for AMS in the province. The committee formed an AMS visiting team comprised of an adult and a paediatric infectious disease specialist, a microbiologist, a pharmacist, an infection prevention and control co-ordinator and a public health medicine specialist. This team conducted visits to healthcare facilities with the aim of monitoring and supporting facility-level initiatives, such as AMS ward rounds, dedicated antimicrobial prescription charts and restrictive prescribing policies to limit the rate of development of antimicrobial resistant organisms, prevent infection and control audits to limit the spread of organisms.

However, apart from these efforts to highlight and promote AMS in the Western Cape, not much is known about the extent and varying models of AMS implementation across the province. A situational analysis of facility-level AMS activities was therefore undertaken to provide a better understanding of such programmes in the Western Cape.

\section{Methods}

\section{Study design}

This study took the form of a self-administered, email questionnaire survey, which was sent to specific cadres of staff at all district, regional and tertiary hospitals in the Western Cape. Hospitals offering specialised services, such as maternity, psychiatric or rehabilitative care, were excluded. Questions were of a quantitative and qualitative nature.

\section{Study sample}

The study sample included hospitals from all 6 health districts of the Western Cape, which comprises one urban (Cape Town Metro) and 5 rural (Cape Winelands, Central Karoo, Eden, Overberg and West Coast) districts. Five hospitals that render exclusively psychiatry, maternity and rehabilitation services were excluded from the study, as they do not routinely prescribe high volumes of antimicrobials.

\section{Data collection}

Email surveys were used to collect data (Appendix A: http://samj. org.za/public/sup/14645-a.pdf). Data were collected from December 2016 to May 2017. Email addresses of the relevant staff were obtained by utilising the search function of the province's central mailing list. To adequately identify and describe the heterogeneous nature of AMS in the province, the survey was addressed to a diverse group of facility staff, including managers, senior clinicians, infectious disease specialists, medical microbiologists, pharmacists, quality assurance personnel and infection prevention and control practitioners. Only one completed survey was required from each facility - respondents were requested to engage with all facility staff members, who were initially contacted by the study team to ensure the responses captured were corroborated as objective by the respondents' colleagues.

Participants were asked to complete a survey form in Microsoft Word (Microsoft Corp., USA) and email the completed form to the principal investigator (SMP). Participants were also given the opportunity to request a telephonic completion of the questionnaire by responding to the email with a telephone number at which they could be contacted.

\section{Data management, analysis and dissemination}

The data obtained from completed surveys were transcribed into a Microsoft Excel (Microsoft Corp., USA) spreadsheet. Descriptive statistics were used to determine the extent and quality of AMS activities. The composition, nature, activities and meeting frequency of stewardship teams, as well as antimicrobial consumption and resistance patterns, were surveyed. The findings and recommendations arising from the study were delivered to the provincial AMS committee and the health impact assessment directorate of the Department of Health, Western Cape Government, to further support the strengthening of AMS activities in the province.

\section{Ethical approval}

Ethical clearance was sought and obtained (ref. no. HREC 802/2016) from the Human Research Ethics Committee, University of Cape Town, and access to the facilities to conduct research was obtained from the Deputy Directorate: Health Research, Department of Health, Western Cape Government. Participation in the study was on a voluntary basis, and no incentives were offered for participation. Information is reported by health district, and individual respondents remain anonymous.

\section{Results \\ AMS committees}

Of the 47 hospitals emailed, 35 responded to the questionnaire, giving a response rate of $74.5 \%$. Of the 35 hospitals that responded, 13 were in the Cape Town metropolitan area (3 tertiary, 1 regional and 9 district), while 22 were in rural districts of the Western Cape (2 regional and 20 district) (Table 1 ).

Approximately half ( 19 of $35 ; 54.3 \%$ ) of these reported active AMS committees (Table 1), which were unevenly distributed between metropolitan (83.3\%) and rural (39.1\%) facilities (Table 1).

\section{Committee compositions, activities and frequency}

In the facilities with established committees, common stewardship activities included antimicrobial prescription chart reviews and audits, AMS ward rounds and antimicrobial restriction policies. Many facilities also provided in-house training of clinical staff on AMS practices (Table 2).

Table 1. Location and referral levels of hospitals and presence of a hospital antimicrobial stewardship committee

\begin{tabular}{llll}
\hline \multirow{2}{*}{ Referral level } & \multicolumn{2}{c}{ Location } & \\
\cline { 2 - 3 } & Metro, $\boldsymbol{n}^{*}$ & Rural, $\boldsymbol{n}^{*}$ & Total, $\boldsymbol{n}^{*}$ \\
\hline Tertiary & 3 & 0 & 3 \\
Regional & 1 & 2 & 3 \\
District & 8 & 21 & 29 \\
Total & 12 & 23 & 35 \\
HAMSC, $n$ (\%) & $10(83.3)$ & $9(39.1)$ & $19(54.3)$ \\
HAMSC $=$ hospital antimicrobial stewardship committee. & \\
*Except where otherwise indicated.
\end{tabular}

Table 2. Antimicrobial stewardship committee activities

\begin{tabular}{ll}
\hline Activity & $\begin{array}{l}\text { Facility } \\
\text { frequency, } \boldsymbol{n}\end{array}$ \\
\hline Antimicrobial prescription charts & 13 \\
Stewardship ward rounds & 13 \\
Antimicrobial restriction policy & 12 \\
Audits & 11 \\
Training & 9 \\
Antibiogram review & 1 \\
Promoting awareness & 1 \\
Policy review & 1 \\
Infection prevention and control training & 1
\end{tabular}


All hospital committees bar one included the hospital pharmacist $(n=18)$, and a majority of committees included infection prevention and control practitioners $(n=15)$, clinical managers $(n=14)$, nurses $(n=14)$ and a varying number of medical specialists, most notably from internal medicine $(n=12)$ and infectious diseases $(n=12)$ (Fig. 1). The results can be misleading, as multiple facilities claimed the same outreach infectious disease sub-specialists as being on their hospital committees; however, there is only a total of 7 in the province. Most committees (12 of 19) reported meeting either monthly or quarterly, with a minority of facilities meeting more frequently. One facility reported holding committee meetings daily.

\section{AMS champions}

Facility-level champions were identified at 20 hospitals. There was diversity in the occupational role of such champions, who were mostly pharmacists $(n=5)$, followed by internal medicine and infectious disease specialists $(n=4)$, family physicians $(n=3)$ and clinical or quality assurance managers $(n=2)$. Certain facilities that did not have AMS committees nevertheless had a designated AMS champion.

Antimicrobial consumption and resistance data

Just less than two-thirds (62.9\%) of responding hospitals reviewed their antimicrobial consumption data on at least a monthly basis. Again, most metropolitan hospitals (12 of 13) reviewed their data, compared with fewer than half of rural hospitals (10 of 22). The source for these data was mainly the pharmaceutical information system of the province - the JAC Pharmacy Management System (a subsidiary of Mediware Information Systems, UK). Other facilities received data from the provincial therapeutics committee of their district.

Most facilities that responded, which had an AMS programme (16 of 22), reviewed their antimicrobial consumption data on a monthly or quarterly basis, with the remainder doing so less frequently.

Just over half (18 of 35) of the facilities did not review AMR patterns. Resistance data reviews were evenly split between metropolitan and rural facilities (6 of 12 metropolitan facilities, and 11 of 23 rural facilities). Most facilities that used resistance data did so on a monthly or quarterly basis. The National Health Laboratory Service (NHLS) was, however, most commonly recorded as the source of these data. The data may refer to individual patient reports of AMR organisms from pathology laboratories. It is assumed that these reports are then collated and reviewed on a regular basis. The reports could also refer to composite resistance patterns published by the NHLS per facility on a regular basis.

All but one facility reported the consumption of antimicrobial guidelines to inform prescribing practice. The most commonly used guidelines include the South African Antibiotic Stewardship Programme (SAASP)'s prescribing guidelines, ${ }^{[10]}$ followed by the Standard Treatment Guidelines and Essential Medicines List for South Africa, ${ }^{[1]}$ produced by the $\mathrm{NDoH}$. It is likely that these documents are not mutually exclusive,

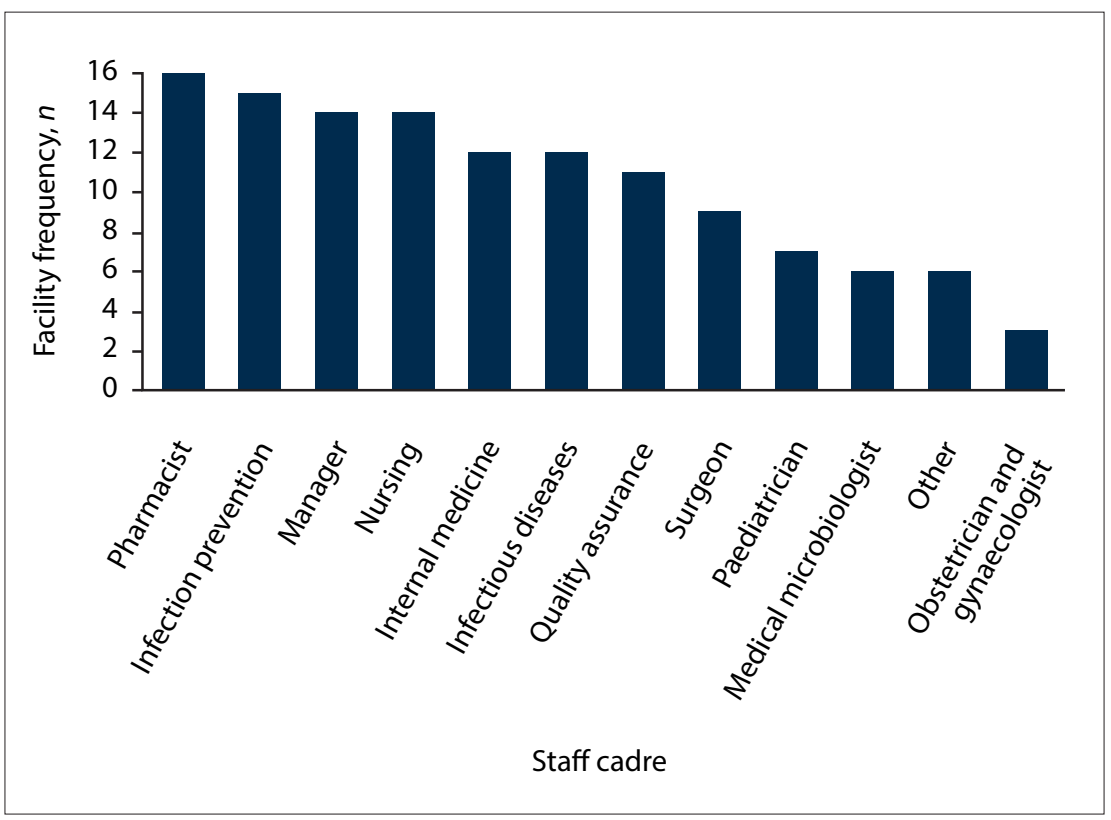

Fig. 1. Antimicrobial stewardship committee composition.

with both being used in most facilities; however, the question was coded to only record one response. Other facilities used hospital-specific guidelines, such as Paarl Regional Hospital's antimicrobial guidelines. A minority of facilities reported using the PACK (Practical Approach to Care Kit) guidelines for primary care, the IMCI (Integrated Management of Childhood Illness), or the provincial medicine code list.

\section{Current outreach and support activities}

In terms of current outreach and support activities, facilities were grouped into four possible categories:

- reception of outreach and support only

- provision of outreach and support only

- neither reception nor provision of outreach and support, or

- reception and provision of outreach and support.

Ten rural and 3 metropolitan facilities reported not receiving support from or providing outreach to other facilities. The 4 facilities that reported providing support only were: 3 tertiary and 1 district level. A large district-level hospital reported receiving and providing outreach and support.

Facilities without full-time pharmacists noted difficulties in implementing guidelines adequately or providing support to other facilities. In these cases, the responsibility for AMS fell on doctors and nurses, who reported being inadequately trained and informed. Clinical staff reported not having enough time to engage in AMS activities due to the volume of the clinical workload.

When facilities reported having provided and received outreach and support, this generally came by way of visiting clinicians from the disciplines of internal medicine, infectious diseases or microbiology, who performed AMS rounds and teaching. While this was appreciated, numerous facilities advised that more frequent and regular contact with such clinicians would be beneficial.

\section{Further support required from the} provincial AMS committee

Most facilities (27 of 35) were aware of the work of the provincial AMS committee. The type of support required by different hospitals included:

\section{Tertiary hospitals}

- Due to their specialised staff complement, outreach was not required by respondents at tertiary facilities; however, sharing of 
best practice with peripheral facilities, as well as an understanding of why certain stewardship practices failed at certain facilities, was noted to be beneficial and able to inform similar initiatives at tertiary level.

\section{Regional hospitals}

- Regional facilities explicitly asked for clinician-driven AMS outreach in the form of regular ward rounds, support and training of staff to be actively rolled out at their facilities and on the primary healthcare platform. At this level, family physicians were identified as the most suitable champions.

\section{District hospitals}

District hospitals asked for a diverse range of interventions in terms of clinical AMS support, as well as help with implementation of setting up teams, protocols and procedures to sustain AMS practices, including:

- assistance with formation and maintenance of AMS committees (especially in small facilities with limited and rotating personnel)

- AMS training and ongoing support for pharmacists, doctors and nurses to increase awareness of the importance of AMS on district hospital and primary healthcare platforms

- dissemination of AMS best practice and pitfalls from other facilities to enable learning opportunities

- a standardised operating procedure and framework for implementation, maintenance and monitoring of AMS in district hospitals

- implementation of norms and standards regarding AMS

- development of hospital antimicrobial guidelines and protocols

- specific training on the rational use of tuberculosis drugs

- implementation of a standardised provincial antimicrobial prescription chart to easily compare and compile dosage, duration, route and patient details

- regular feedback on provincial and facility antimicrobial consumption statistics and trends to improve facility learning and accountability

- immediate verbal or other direct communication with heads of department or facility managers regarding important changes in resistance patterns.

\section{Discussion}

This study demonstrates variation in AMS activities at district, regional and tertiary hospitals in the Cape metropolitan and rural districts. Consistent with the limited local research available, ${ }^{[5,12]}$ AMS activities, committee composition and support varied by location and across the level of care.

Support is particularly required in rural districts, where facilitylevel AMS committees still need to be established. Hospitals without committees were generally smaller facilities with smaller staff complements, or district-level hospitals that provide in-patient convalescence and treatment for tuberculosis.

Although there were common themes across metropolitan and rural facilities, rural respondents also drew attention to the difficulties inherent in arranging AMS activities in smaller, more remote hospitals with a large proportion of frequently rotating junior staff, such as community service medical officers. Respondents therefore requested assistance with the logistics of AMS activities: setting up and guiding the functioning of AMS committees, assisting with the clinical governance role of such committees, and helping with ensuring sustainability of these committees, in the context of a relatively small and constantly rotating workforce. Some respondents noted that there was a paucity of necessary staff to drive AMS activities, as certain rural areas had entire health districts without any pharmacists, in which case AMS became the responsibility of frontline nurses and doctors, who felt ill-prepared to perform the task.

Medical specialists have successfully led AMS programmes, particularly in regional and tertiary-level hospitals. ${ }^{[12]}$ Given that most of the regional and tertiary hospitals are located in the Cape metropole, it is unsurprising that the majority of AMS committees were established in metropolitan facilities.

We found that AMS activities were conducted at all facilities, independent of the establishment of formal AMS committees. This highlights the importance of a committed team and suggests that AMS champions at facility level may be an under-recognised resource, which if utilised optimally, could lead to AMS success.

Pharmacists and infectious disease practitioners served on most committees. However, the skills and experience mix of committees varied. The skills mix has been shown to determine the strategy and activities of AMS. ${ }^{[12]}$ Our study did not explore roles, but Brink et al. ${ }^{[6]}$ found that roles evolved in line with AMS demands. At district hospital level, roles of members serving on AMS committees were less specific than those at regional and tertiary level due to smaller teams. Neither model was deemed superior. ${ }^{\left[{ }^{5}\right]}$ However, a multi-skilled team that displays commitment to leading AMS culture change is imperative. ${ }^{[13]}$

AMS activities included antimicrobial prescription chart reviews and audits, AMS ward rounds and antimicrobial restriction policies. Antimicrobial consumption data and AMR patterns, key elements of an AMS programme, were conducted by only half of the existing committees, emphasising the need for clear objectives of facilitybased AMS. Von Pressentin et al..$^{[12]}$ further suggest that data support is needed to ensure effective metrics for surveillance at facility level. The exploration of information management or data support as a potential barrier was beyond the scope of the objectives of this study.

The question of whether antimicrobial consumption data are used in facilities, had a few obscure responses, including ward rounds, patient folders or the NHLS. These answers raise questions as to individual respondents' understanding of the term data, with the abovementioned responses indicating the use of single person-toperson antimicrobial consumption reviews, rather than the use of a comprehensive, aggregated database of antimicrobial consumption collected and reviewed over a set period of time to inform and adapt prescribing behaviour.

Reports of committees meeting more frequently than once a month seem to be highly unlikely, and may indicate confusion between the roles of clinical teams engaging in AMS activities, such as antibiotic review ward rounds, and formal, agenda-driven, chaired meetings of hospital AMS committees, as described in the National Guidelines on Implementation of the Antimicrobial Strategy in South Africa. ${ }^{[9]}$

Most metropolitan hospitals received or provided AMS outreach support, while few rural hospitals received or provided such support. Sub-optimal support is a common challenge at facility level, ${ }^{[13]}$ and it is recommended that provincial committees ensure that support functions and roles are communicated ${ }^{[13]}$ in line with the view of Begg et al.. ${ }^{[13]}$ underscoring co-operation and collaboration as key to successful AMS programmes.

\section{Study limitations}

The scope of this study was limited to describing AMS efforts undertaken in the Western Cape and to explore opportunities and impediments to the development of facility-level AMS programmes. Further limitations of this study are the exclusion of information 
on baseline antimicrobial use data and antimicrobial and resource availability. Furthermore, research on veterinary services, information management, community health centres, clinics and district and provincial stewardship committees would add value to the current body of knowledge, as well as a review of enablers and barriers to AMS committee formation and maintenance.

Respondents were requested to engage with relevant staff at their respective facilities, and the study questionnaire was sent to multiple staff members. However, due to the data collection method used in this study, it was not possible to assess the level of engagement with the questionnaire at different hospitals.

Nevertheless, the study has been imperative in providing insight regarding the status of AMS efforts in the Western Cape. The findings could be used to revise AMS activities at provincial and facility level.

Future studies should investigate the role of information management and the effectiveness of outreach support and other tools to strengthen AMS.

\section{Declaration. None.}

Acknowledgements. We thank the provincial AMS committee and visiting team for their oversight and guidance. We also thank all facilities with functioning AMS committees and activities, and the facilities that participated in the study.

Author contributions. The study protocol was developed by SS, data collection and analysis were performed by SMP, report writing and compilation of the discussion were co-ordinated by JLW, and the study was supervised by BW and M-AD.

Funding. None.

Conflicts of interest. At the time of preparing the manuscript, the authors were salaried employees of the Department of Health, Western Cape Government. BW occupied a provincial public health medicine specialist post between 2015 and 2019. Antibiotic stewardship support formed part of the responsibilities of this position. SS, SMP and JLW were provincial public health medicine registrars with shared responsibilities to antibiotic stewardship activities between 2015 and 2017.

1. World Health Organization. Urgent action necessary to safeguard drug treatments. 2011. www.who. int/mediacentre/news/releases/2011/whd_20110406/en/(accessed 5 March 2021).

2. Antimicrobial resistance: Tackling a crisis for the health and wealth of nations. 2014. http://www. Antimicrobial resistance: Tackling a crisis for the health and wealth of nations. 2014. http://www.
jpiamr.eu/wp-content/uploads/2014/12/AMR-Review-Paper-Tackling-a-crisis-for-the-health-andwealth-of-nations_1-2.pdf (accessed 5 March 2021).

3. Sartelli M, Labricicciosa FM, Barbadoro P, Pagani L, Ansaloni L, Brink AJ. The Global Alliance for Infections in Surgery: Defining a model for antimicrobial stewardship - results from an international cross-sectional survey. World J Emerg Surg 2017;12:34. https://doi.org/10.1186/s13017-017-0145-2 4. Davey P, Brown E, Charani E, et al. Interventions to improve antibiotic prescribing practices for hospital inpatients. Cochrane Database Syst Rev 2013. https://doi.org/10.1002/14651858. CD003543.pub3

5. Boyles TH, Naicker V, Rawoot N, Raubenheimer PJ, Eick B, Mendelson M. Sustained reduction in antibiotic consumption in a South African public sector hospital: Four-year outcomes from the Groote Schuur Hospital antibiotic stewardship programme. S Afr Med J 2017;107(2):115-118. https://doi. org/10.7196/SAMJ.2017.v107i2.12067

6. Brink AJ, Messina AP, Feldman C, et al. Antimicrobial stewardship across 47 South African hospitals: An implementation study. Lancet Infect Dis 2016;16(9):1017-1025. https://doi.org/10.1016/S14733099(16)30012-3

7. Boyles TH, Whitelaw A, Bamford C, et al. Antibiotic stewardship ward rounds and a dedicated prescription chart reduce antibiotic consumption and pharmacy costs without affecting inpatient mortality or re-admission rates. PLoS ONE 2013;8(12):e79747. https://doi.org/10.1371/journal. pone. 0079747

8. Departments of Health and Agriculture, Forestry and Fisheries. Antimicrobial Resistance National Strategy Framework 2018 - 2024. Pretoria: National Department of Health, 2017.

9. National Department of Health. The National Guidelines on Implementation of the Antimicrobia Strategy in South Africa: One Health Approach and Governance. Pretoria: NDoH, 2017.

10. Wasserman S, Boyles T, Mendelson M. The South African Antibiotic Stewardship Programme. A Pocket Guide to Antibiotic Prescribing for Adults in South Africa. 2014. https://www.fidssa.co.za/ Content/Documents/SAASP_Antibiotic_Guidelines_2015.pdf (accessed 28 September 2020).

11. National Department of Health. Standard Treatment Guidelines and Essential Medicines List for South Africa: Hospital Level, Adults. Pretoria: NDoH, 2019.

12. Von Pressentin KB, Swanepoel H, Opie JJ, Jenkins LS. Antimicrobial stewardship in rural districts of South Africa: Growing a positive culture. S Afr Fam Pract 2019;61(6):276-281. https://doi.org/10.108 South Africa: Growing a p

13. Begg K, Mamdoo P, Dudley L, Andrews G, Engelbrecht J, Lebese L. South African Health Review: Development of a National Strategic Framework for a High-quality Health System in South Africa. Durban: Health Systems Trust, 2018.

Accepted 5 December 2020. 